\title{
Addressing Communication and Rumination in Physician/Patient Interactions: Implications for Treatment Compliance
}

\author{
Ralph Dell'Aquila*
}

Psychologist, 180 Boyden Ave, Maplewood, NJ 07039, USA

\begin{abstract}
Effective communication between physicians and patients is vital and necessary for proper medical care. The problem is that there are times in which misunderstandings can take place. Because of the nature of medical care and conditions, the effects of miscommunication can have devastating effects especially for those who have co-occurring medical conditions and mental health disorders such as depression. Thus, the article outlines the following: How to apply empathic interventions to build therapeutic rapport, identify rumination in communication with patients and manage rumination by promoting psychological flexibility.
\end{abstract}

Keywords: Therapeutic rapport; Empathy; Rumination; Physician

\section{Introduction}

According to the World Health Organization (WHO), depression is the leading cause of disability worldwide with an estimated 300 million people affected each year [1]. Physicians see patients whose physical symptoms have mental health components and are often on the front line in assessing those who have symptoms of depression. According to clinicians who specialize in cognitive therapy, non- functional thoughts about the self, world and future lead to and sustain feelings of depression; Beck termed this phenomenon as the classic triad [2].

One such example of non-functional thoughts is rumination which is defined as misattribution of facts, hopelessness and repetitious/nonproductive thoughts [3]. People who use rumination tend to be fixated on the problem and feelings associated with it rather than employing an active problem solving strategy [4]. The challenge remains in terms of how to build a solid therapeutic rapport with patients so that the cognitive techniques can be personalized. Without a "buy in" from patients, the application of cognitive strategies to address depression will be limited. One of the best ways to gauge motivation is through listening to patients and examining the various aspects of their lives.

One of the first things that healthcare professionals learn is to take a history of patients. This is important because it enables us to see the presenting problem within a specific context and in so doing, a tailored approach can be applied to help the individual. In order for the history and consequent interventions/compliance to be effective, people have to feel comfortable with practitioners. In addition to a mental status exam, key information could include the following: duration of symptoms, history of mental health/addiction issues, presence of pain/ chronic conditions, important life events/developmental milestones, current family structure/social support, trauma/recent deaths, occupational status and family history.

Patients sense a physician's empathy in the verbal and non-verbal interactions; empathic interactions have been shown to lead towards better clinical outcomes for patients [5]. Fostering empathy promotes collaboration, support and promotes positive exchange of salient information pertaining to a patient's life [6,7]. Rogers defined empathy as the willingness to view the client's world through his or her eyes as if you were that client and not forgetting the as if. Empathy is conveyed by active listening techniques that show patients that physicians are actively engaged in the information patients are sharing [8]. Hashim discussed the importance of being present by the use of active listening which involves open ended questions and responding to patient statements by capturing the emotional content of what patients reveal [9]. The takeaway here is that building a therapeutic rapport via empathic interventions can help a patient feel more comfortable to open up about specific challenges he/she faces.

When considering the "presenting problem", it is important to examine the personal expectations of patients who see healthcare providers. As practitioners listen to the patient tell his/her story, they can evaluate expectations which form the assumptions /foundations of the thought processes as they relate to depression and related mental health symptoms. It is also good to see if the thoughts are within the realm of reality. Special consideration should be made for those who are grieving a recent death.

Rumination is a thought process that causes people to respond to internal distress by obsessively focusing on the external causes of one's depressive symptoms rather than employing active strategies to examine the circumstances and personal resources to find a resolution [10]. Over time, non-functional thought processes can lead to increased depression, anxiety, increase substance misuse/relapse and can exacerbate physical pain such as headaches, stomach issues and back pain.

Rumination is composed of four factors including: 1. Problem focused/problem solving difficulties which leads to internal stress, 2. Counterfactual thinking- visualizing and focusing on real/imagined scenarios involving regret, shame/guilt, 3. Repetitious thoughtspersistent/automatic obsessive non-functional thoughts and 4 . Anticipatory Thoughts- worrying about future events/outcomes [11]. While not everyone will exhibit all four simultaneously, the conceptualization is useful as physicians assess a patient's mental health status and evaluate present coping resources.

*Corresponding author: Ralph Dell'Aquila, Psychologist, 180 Boyden Ave Maplewood, NJ 07039, USA, Tel: 9733786012; E-mail: dottralph@yahoo.com

Received December 04, 2018; Accepted March 12, 2019; Published March 19 2019

Citation: Dell'Aquila R (2019) Addressing Communication and Rumination in Physician/Patient Interactions: Implications for Treatment Compliance. J Pat Care 5: 145. doi: 10.4172/2573-4598.1000145

Copyright: $\odot 2019$ Dell'Aquila R. This is an open-access article distributed unde the terms of the Creative Commons Attribution License, which permits unrestricted use, distribution, and reproduction in any medium, provided the original author and source are credited. 
While the rumination thought process is generally seen as individual, the phenomenon can manifest itself within the context of a dyad relationship. Co-rumination occurs when two people obsessively dwell on negative aspects of an issue rather than work towards finding a viable solution or resolution to the presenting issue [12]. In this context, it is helpful to assess whether patients have adequate positive social support or if the patient is surrounded by unhelpful communication which may contribute to pathology.

Assessment of rumination is helpful because it can also identify treatment compliance issues in terms of patient ambivalence towards change. Merino, Semra and Ferreiro found that brooding was positively correlated with depressive thoughts while worry was more linked to anxiety [13]. It should be kept in mind that patients can exhibit a combination of anxiety and depression. Although rumination and worry are correlated, they differ in the following way: 1. rumination is focused on the past/people tend to view event as as certain, uncontrollable and they seek insight, 2 . worry is focused on the future, views events as uncertain/potentially controllable and they try to anticipate/prepare for the defined threat [14].

A main strategy to use in reducing rumination is to promote reprocessing of the information. Psychological flexibility includes the following: 1 . Recognize and adapt to a variety of situational demands and 2. Shift mindset about the situation and adjust behavior/thoughts accordingly [15]. A key part of working towards psychological flexibility involves re-appraisal of non-functional thinking patterns. The collaborative empiricism of Beck et. al tests the thoughts of patients by looking at the evidence of the thoughts and practitioner/patient discuss ways to develop more adaptive thought processes [2].

The ability to think of a situation from a different perspective may seem simple but it can make a big difference in how people face personal challenges. The monitoring of rumination and non-helpful thoughts is vital for patients to do when they are also out of the office between visits. Patience in working with patients who ruminate is important because there are many reasons why these non-helpful beliefs may persist.

The ability of physicians to convey empathy is important in addressing short term and long term concerns of patients. This is because empathic interventions promote understanding the World, as the patient sees/exists in it. Hashim spoke about the value of responding to verbal and non-verbal cues from the patient within the context of also taking a medical history [9]. This can be valuable as it helps physicians pinpoint the salient themes and the level of motivation, on the part of patients, can be assessed.

Jeffrey defined healthcare professional/patient empathy as having the following: an emotional two way connection, desire to understand patient's specific situation through their patient's perspective, healthcare professional seeing patient viewpoint in managing condition and active response to help patient through skilled interventions [16]. While Bloom agrees with empathy in the context of purporting to understand other people and their thoughts, he expressed concern about clinicians who try to feel what patients feel as this can affect objectivity as people tend to have more empathy for people like themselves and proposed rational compassion instead of empathy [17]. In the application of empathy as a clinical skill set, it is possible to strike a balance between cognitive and emotional empathy.

Reiss discussed how cognitive empathy can be effective in cases where medical professionals are helping those who are culturally different [18]. Empathy incorporates cognitive and emotional components to help make sense of a patient's experience while at the same time, recognizing the distinctions between the self and patient [19]. Rogers defined empathy as the willingness to view the client's world through his or her eyes as if you were that client and not forgetting the as if [8].

Those who reported high levels of rumination had higher levels of anxiety, depression, negative coping styles and lower ratings of selfefficacy and optimism [20,21]. The identification of how patients see themselves in terms of self-efficacy can influence treatment compliance and wellness. For many health conditions, medication alone will not be enough to keep a person on the road to wellness. Personal elements such as a patient feeling ambivalent, embarrassed, cultural differences or a patient's past negative experiences in healthcare can affect health and wellness choices between office visits. By attending to a patient's perspective, healthcare professionals can optimize medical interventions.

\section{Discussion and Conclusion}

Those who engage in rumination may have a more difficult time in managing feelings such as depression or anxiety because they ascribe external factors to their symptoms. Clinician attention to how personal relationships and context can perpetuate rumination can be explored $[2,11]$. Lastly, psychological flexibility via collaborative empiricism can help patients to re-examine non-functional thoughts and reframe them into more adaptive ones.

\section{References}

1. World Health Organization Mental Health. Mental disorders.

2. Beck Aaron T, Rush AJ, Shaw BF, Emery G (1987) Cognitive therapy of depression. Guilford Press: New York.

3. Lyubormirsky S, Nolen-Hoeksema S (1995) Effects of self-focused rumination on negative thinking and interpersonal problem solving. J Pers Soc Psychol 69: $176-190$

4. Nolen-Hocksema S, Wisco BE, Lyubomirsky S (2008) Rethinking rumination. Perspect Psychol Sci 3: 400-424.

5. Hojat M (2009) Ten approaches for enhancing empathy in health and human services culture. J Health Hum Serv Adm 31: 412-450.

6. Kurtz S, Silverman J, Draper J (2005) Teaching and learning communication skills in medicine. 2nd ed. Oxford: Radcliffe Publishing.

7. Larson EB, Yao X (2005) Clinical empathy as emotional labor in the patient physician relationship. JAMA 293: 1100-1106.

8. Rogers CR (1957) The necessary and sufficient conditions of therapeutic personality change. J Consult Psychol 21: 95-103.

9. Hashim MJ (2017) Patient centered communication: Basic skills. Am Fam Physician 95: 29-34.

10. Nolen Hoeksema S (1991) Responses to depression and their effects on the duration of depressive episodes. J Abnorm Psychol 100: 569-582.

11. Tanner A, Voon D, Hasking P, Martin G (2012) Underlying structure of ruminative thinking: Factor analysis of the ruminative thought style questionnaire 37: 633-646.

12. Balsamo M, Carlucci C, Sergi MR (2016) The mediating role of early maladaptive schemas in the relation between co-rumination and depression in young adults.

13. Merino Semra C, Ferreiro F (2016) Are worry and rumination specific pathways linking neuroticism and symptoms of anxiety and depression in patients with generalized anxiety disorder, major depressive disorder and mixed anxiety depressive disorder.

14. Lyubomirsky S, Layous K, Chancellor J, Nelson SK (2015) Thinking about rumination: The scholarly contributions and intellectual legacy of Susan NolenHoeksema. Annu Rev Clin Psychol 11: 1-22. 
Citation: Dell'Aquila R (2019) Addressing Communication and Rumination in Physician/Patient Interactions: Implications for Treatment Compliance. J Pat Care 5: 145. doi: 10.4172/2573-4598.1000145

Page 3 of 3

15. Kashdan TB, Rottenberg J (2010) Psychological flexibility as a fundamental aspect of health. Clin Psychol Rev 30: 865-878.

16. Jeffrey $D$ (2016) Clarifying empathy: The first step to more humane clinical care. Br J Gen Pract 66: e143-e145.

17. Bloom $P$ (2016) Against empathy: The case for rational compassion. Harper Collins: New York.

18. Coplan A, Goldie P (2011) Empathy philosophical and psychological perspectives. Oxford: Oxford University Press.

19. Reiss $H$ (2017) The science of empathy. J Pat Exper.

20. Jeffrey D (2012) Empathy, sympathy and compassion in healthcare: Is there a problem? Is there a difference? Does it matter? J Royal Soc Med 109: 446452.

21. Malin K, Littlejohn GO (2015) Rumination modulates stress and othe psychological processes in firbromyalgia 2015. Eur J Rheumatol 4: 143-148. 